\title{
Training Implications for Firefighters through objective Measurement of the Physiological Demands of Firefighter Job Tasks
}

\author{
Dierdra K Bycura*, Christopher P Repka, Anthony C Santos and Nanette V Lopez \\ Department of Health Sciences, Northern Arizona University, USA \\ *Corresponding author: Dierdra K Bycura, Department of Health Sciences, Northern Arizona University, USA. \\ To Cite This Article: Dierdra K Bycura. Training Implications for Firefighters through objective Measurement of the Physiological Demands of \\ Firefighter Job Tasks. Am J Biomed Sci \& Res. 2019 - 3(5). AJBSR.MS.ID.000715. DOI: 10.34297/AJBSR.2019.03.000715
}

Received: June 29, 2019 | Published: July 08, 2019

\begin{abstract}
The purpose of this study was to describe the direct measurement of physiological demands of firefighter job tasks within a modified Candidate Physical Abilities Test (CPAT) broken out by specific task. Twenty male firefighters from a large municipal fire department in the southwest United States participated in the study. To directly measure cardiorespiratory demands of firefighting job tasks, the study incorporated the measurement of individual firefighter physiological demands on four different physiological variables (VO2, Heart Rate-HR, Respiratory Exchange Ratio-RER, Energy Expenditure per Minute-EEM). These four variables were measured during participant completion of a modified physical protocol for firefighters (CPAT); 8 tasks repeated for 15 minutes in duration at baseline, 6 weeks post-baseline, and 14 weeks post-baseline, using the Cosmed K4b2 portable metabolic system. Tukey's HSD comparison test was performed to compare the differences between responses of each task ( $p \leq 0.05)$. Results demonstrated significant differences between some tasks. VO2 requirement to complete the tasks increased from baseline to 14 weeks post-baseline while RER decreased. Knowledge of physiological work required of specific and accumulated firefighter job tasks could inform type of training, timing and fitness ability needed to safely perform job tasks and directly target fitness maintenance for the job.
\end{abstract}

Keywords: Firefighter job tasks; Tactical strength; Conditioning; Functional training

Abbrevations: CPAT: Candidate Physical Ability Test; RER: Respiratory Exchange Ratio; HR: Heart Rate; EEM: Energy Expenditure per Minute Tukey's; HSD: Honestly Significant Difference; BMI: Body Mass Index; CVD: Cardiovascular Disease

\section{Introduction}

It is well known that firefighting is physically demanding occupation often requiring high intensity work in a continuous or intermittent manner. Additionally, other aspects of the job such as long shift work, reported sleep deprivation and the necessity to rapidly respond to emergency calls place firefighters at even greater health risk [1]. It is important to acknowledge and evaluate the various demands of career firefighters to inform exercise training design to directly target the observed health risks of persons in this profession. Previous studies examining large cohorts of career firefighters report specific health risks that are associated with the job, such as the documented decrease in cardiorespiratory fitness, increase in body mass index (BMI), and higher incidence of occupational injury among firefighters as they age [2-6]. Korre et al. [7] further supported the link between cardiovascular disease (CVD) risk and BMI, specifically the correlation between increased BMI and left ventricular mass increase in this population of which both are indications of CVD risk. These studies support the need for exercise training that addresses cardiovascular endurance for heart health and body weight reduction in this population.

Regarding type of exercise training and propensity for injury, Lentz et al. [8], conducted a systematic review examining the relationship between physical fitness and occupational injury in emergency responders and found a consistent trend between lower levels of aerobic fitness and increase in injury risk. They concluded that future research should focus on interventions that address both aerobic endurance and components of fitness such as muscular strength, balance, and agility to determine the optimal level of fitness conducive to effective job performance and injury prevention. While aerobic endurance and muscular strength are important health-related components of fitness needed for successful job performance in first responders, skill related 
components of fitness (balance, agility, speed, power, coordination and reaction time) are also important requirements for safe job performance in this population.

Recent work conducted by Gerstner et al. $[9,10]$ discussed the age-related changes in muscle quality required for peak torque in career firefighters. The ability of firefighters to produce maximal strength at both slow and fast velocities is necessary for successful, injury free completion of job required tasks. These authors reported a decrease in peak torque and an increase in intramuscular fat infiltration both of which are associated with metabolic and cardiovascular risk factors. They conclude by suggesting the implementation of tactical strength and conditioning exercise training to target performance requiring strength at fast velocities. Persons interested in becoming a career firefighter are oftentimes required to demonstrate their physical capability to perform firefighter job tasks prior to getting hired for the job. Men and women interested in this job are usually young adults in peak physical condition prepared to be tested on their fitness and specific aptitude for the required job tasks. Many fire departments have adopted the Candidate Physical Ability Test (CPAT) to determine candidate physical readiness for the profession.

The CPAT was developed in 1997 by the Fire Service Joint Labor-Management Task Force Technical Committee to measure critical skills of firefighting job tasks. This committee conducted a job analysis and administered job task surveys to more than 1000 fire fighters across ten departments, to ascertain the necessary skills needed to perform firefighter job tasks. The aim was to develop an authentic and valid evaluation tool to ensure that all fire fighter candidates had the physical ability to complete critical tasks effectively and safely. Currently, the CPAT is approved by the International Association of Fire Chiefs and the International Association of Fire Fighters as an appropriate pre-employment physical test to determine physical capabilities of firefighter candidates [11]. The CPAT is comprised of a series of eight firefighting tasks completed for time with an established a pass/ fail time of 10 minutes and 20 seconds. However, when on a fire or emergency scene, firefighters are often required to work for longer periods of time and pace themselves rather than work at their maximum level for 10 minutes. We are just beginning to investigate the cumulative physiological effect or metabolic demand of firefighter job tasks when performed for a prolonged period rather than for a shorter time at maximal intensity [1].

It has been reported that firefighters often face health challenges that can be related to the job such as increased incidence of cardiovascular events over time while the job task demand remains high [2-6]. Moreover, we know that as a part of the natural aging process, there is an age-related decline in cardiorespiratory fitness [12]. There is also literature supporting a decrease in regular physical activity among firefighters as they age $[2,13,14]$. The purpose of this study was to further describe the direct measurement of physiological demands of firefighter job tasks within a modified CPAT course broken out by specific task. Knowledge of physiological work required of specific and accumulated firefighter job tasks could inform type of training, timing and fitness ability needed to safely perform job tasks and directly target fitness maintenance for the job.

\section{Materials and Methods}

\section{Experimental approach to the problem}

The National Fire Protection Association recommends a minimum cardiorespiratory fitness capacity of 12 metabolic equivalents (METS), which is approximately $42 \mathrm{~mL} \cdot \mathrm{kg}-1 \cdot \mathrm{min}-1$ VO2 for firefighters to adequately and safely perform firefighter job tasks [15]. It is important to note that the $42 \mathrm{~mL} \cdot \mathrm{kg}-1 \cdot \mathrm{min}-1 \mathrm{VO} 2$ is a peak value not a value that firefighters normally maintain for a prolonged period of time.

Previously, our team examined physiological requirements of completing a modified CPAT course in a continuous manner [1]. For that study, the modified CPAT was not used as a full effort test for completion time. The test was used to mimic a typical series of job specific tasks. The firefighters in that particular municipal fire department were required to complete a yearly VO2 max test as a part of their job. We took their reported value above $42 \mathrm{~mL} \cdot \mathrm{kg}-1$ -min -1 V02 as "fit for the job" and were really asking the question, "What is the VO2 required to do the 8 job related tasks in a steady state manner over 15 minutes time and could the VO2 required of the typical work move in a favorable direction (e.g. would heart rate be lower on the total or specific task over time)?". The current methodology expands scientific knowledge by further describing the physiological demand (HR, VO2 and RER) by breaking out each individual CPAT task as compared to the number/length of other CPAT tasks. This is one of very few studies to use an actual direct measure of the firefighter job tasks rather than MET calculations, field or treadmill testing to determine the work of the job $[2,6,16]$.

\section{Participants}

Twenty firefighters from a large municipal fire department in the southwest United States participated in the study. Participants ranged in age from 33 to 57 years old $(M=44.10, S D=6.43)$ and averaged 16 years on the job $(M=16.6, S D=6.62)$. Both male and female firefighters with 8 or more years on the job were recruited $(\mathrm{N}=22)$ although only males chose to participate in the study. Two participants dropped out for personal reasons after the first round of testing. No additional exclusion or inclusion criteria were included although after inquiry of the department under study, it was noted that no participants had been on light duty in the past year prior to participating in the study. A signed letter of support from the fire chief of the fire department under study was obtained. The study was approved by the Institutional Review Board at the sponsoring University. All participants signed the approved informed consent prior to taking part in the study.

\section{Procedures}

To directly measure cardiorespiratory demands of firefighting job tasks, the study incorporated the direct measurement of individual firefighter physiological demand on four different physiological variables (VO2, Heart Rate-HR, Respiratory Exchange Ratio-RER, Energy Expenditure per Minute-EEM). These four variables were measured during participant completion of a 
modified version of a standardized physical protocol for firefighters (CPAT- Table 1); 8 tasks repeated for 15 minutes in duration at baseline, 6 weeks post-baseline, and 14 weeks post-baseline, using the Cosmed K4b2 portable metabolic system. Participants were instructed to work at an even pace over the 15-minute time frame in order to elicit a steady state aerobic response to typical firefighter job tasks. After completion of task 8, they re-started task 1 and continued completing tasks until they finished 15 minutes of total work. However, few firefighters completed more than 8 tasks during the 15-minute time frame. Therefore, to adequately perform statistical analyses, VO2, HR, RER, and EEM values were averaged across the first set of 8 tasks for each participant at each time point. The Cosmed K4b2 portable metabolic system was calibrated per system protocols (turbine, room air and reference gas) prior to use on the first participant each day of testing. The specific course used during the study was modified to accommodate what was available at the site training center yet closely mimic the official CPAT course (see Table 1 for modifications to CPAT). This course was held outdoors, and ambient temperature was monitored during each testing session ranging between 63- and 82-degrees Fahrenheit. Participants' wore their full turnout gear which included turnout pants, coat, boots, and helmet although did not wear their selfcontained breathing apparatus. Weight (lbs.) was measured with and without prior to performing the physical tasks at each testing time point (Table 1).

Table 1: CPAT Course Task Descriptions and Modifications. [1].

\begin{tabular}{|c|c|c|}
\hline CPAT Task & Official Course & Modified Course \\
\hline Task 1: Stair Climb & $\begin{array}{l}20 \text { second warm-up at } 50 \text { steps per minute precedes } 3 \\
\text { minutes of stair climbing at } 60 \text { steps per minute. Candi- } \\
\text { dates carry an additional } 25 \text { lbs. in event. }\end{array}$ & $\begin{array}{l}\text { Participants walked up and down a stairwell for } 3 \\
\text { minutes at a pace of } 60 \text { steps per minute. Participants } \\
\text { carried } 25 \mathrm{lb} \text {. hose bundle over right shoulder. }\end{array}$ \\
\hline Task 2: Hose Drag & $\begin{array}{l}\text { With } 8 \text { feet }\left(8^{\prime}\right) \text { of hose slung over shoulder, run } 75 \text { feet } \\
\left(75^{\prime}\right) \text { around drum, make a } 90^{\circ} \text { turn, and run an additional } \\
25 \text { feet }\left(25^{\prime}\right) \text {. Candidates then crouch down on one knee } \\
\text { and pull } 50 \text { feet }\left(50^{\prime}\right) \text { of hose. }\end{array}$ & Replicated event exactly. \\
\hline Task 3: Equipment Carry & $\begin{array}{l}\text { Carry circular saw and chain saw } 75^{\prime} \text { to cone and } 75^{\prime} \text { back } \\
\text { to starting position. }\end{array}$ & $\begin{array}{l}\text { Replicated event using two } 25 \mathrm{lb} \text {. weight plates to simu- } \\
\text { late both saws. }\end{array}$ \\
\hline $\begin{array}{l}\text { Task 4: Ladder Raise and } \\
\text { Extension }\end{array}$ & $\begin{array}{l}\left.\text { Raise a } 24 \text { foot ( } 24^{\prime}\right) \text { extension ladder from the ground to } \\
\text { wall, then extend a } 24^{\prime} \text { pre-positioned extension ladder } \\
\text { secured to wall. }\end{array}$ & Replicated event exactly. \\
\hline Task 5: Forcier Entry & $\begin{array}{l}\text { Strike a fixed measuring device using a } 10 \mathrm{lb} \text {. sledgeham- } \\
\text { mer until the buzzer sounds. }\end{array}$ & $\begin{array}{l}\text { Participants struck a car quarter panel } 10 \text { times from } \\
\text { waist height using a } 10 \mathrm{lb} \text {. sledgehammer. }\end{array}$ \\
\hline Task 6: Search & $\begin{array}{l}\text { Crawl through darkened } 3 \text { foot }\left(3^{\prime}\right) \text { high, } 4 \text { foot }\left(4^{\prime}\right) \text { wide, } \\
\text { and } 64 \text { foot }\left(64^{\prime}\right) \text { long confined space. Candidates make } \\
\text { two } 90^{\circ} \text { turns and circumvent interior obstacles. }\end{array}$ & $\begin{array}{l}\text { Replicated event through darkened burn room while } \\
\text { performing a left wall search. }\end{array}$ \\
\hline Task 7: Rescue & $\begin{array}{l}\text { Drag } 165 \mathrm{lb} \text {. mannequin } 35 \text { feet }\left(35^{\prime}\right) \text {, around drum, and } \\
35^{\prime} \text { back to starting position. }\end{array}$ & $\begin{array}{c}\text { Replicated event with } 170 \text { lb. hose dummy dragged over } \\
\text { asphalt. }\end{array}$ \\
\hline Task 8: Ceiling Breach and Pull & $\begin{array}{l}\text { Candidates use a } 6 \text {-foot ( } 6^{\prime} \text { ) pike pole to press a } 60 \mathrm{lb} \text {. } \\
\text { hinged door up } 3 \text { times. Candidates then use pike poll to } \\
\text { pull an } 80 \mathrm{lb} \text {. hinged door down } 5 \text { times. This sequence is } \\
\text { repeated } 4 \text { times. }\end{array}$ & $\begin{array}{c}\text { Participants raised a } 45 \mathrm{lb} \text {. Olympic barbell, simulating } \\
\text { the pike pole, three times overhead. Participants then } \\
\text { grasped the rope of the fixed } 24^{\prime} \text { extension ladder as } \\
\text { high up as they could and pulled down } 5 \text { times. This } \\
\text { sequence was repeated } 4 \text { times. }\end{array}$ \\
\hline
\end{tabular}

${ }^{*}$ Candidates wear $50 \mathrm{lb}$. weighted vests and walk 85 feet (85') between events in actual CPAT course. Participants in study wore Cosmed K4b2 unit (2.7 lbs.), FF gear without air bottle (weight in gear was measured), and also walked 85 ' between events.

\section{Statistical Analysis}

Table 2: Demographic Characteristics and Descriptive at Baseline.

\begin{tabular}{|c|c|}
\hline Characteristic & Total Sample (N=20) M (SD) \\
\hline \multicolumn{2}{|c|}{ Demographic Characteristics } \\
\hline Age (yrs, at Baseline) & $44.10(6.43)$ \\
\hline Weight (lb) & $205.03(24.36)$ \\
\hline Height (in) & $71.10(2.57)$ \\
\hline Weight in gear (lb) & $228.91(25.58)$ \\
\hline Years on the Job & $16.6(6.62)$ \\
\hline BMI $\left(\mathrm{kg} / \mathrm{m}^{2}\right)$ & $28.63(3.59)$ \\
\hline \multicolumn{2}{|c|}{ Dependent Variables at Baseline } \\
\hline $\mathrm{VO}_{2}(\mathrm{~mL} / \mathrm{kg} / \mathrm{min})$ & $25.20(3.34)$ \\
\hline Heart Rate (beats/min) & $157.17(10.78)$ \\
\hline
\end{tabular}




\begin{tabular}{|c|c|}
\hline Respiratory Exchange Ratio & $1.18(0.07)$ \\
\hline Energy Expenditure per Minute (EEM) (kcal/min) & $13.75(1.55)$ \\
\hline \multicolumn{1}{|c|}{ Dependent Variables at Midpoint } \\
\hline $\mathrm{VO}_{2}(\mathrm{~mL} / \mathrm{kg} / \mathrm{min})$ & $25.87(3.68)$ \\
\hline Heart Rate (beats/min) & $159.54(10.78)$ \\
\hline Respiratory Exchange Ratio & $1.17(0.07)$ \\
\hline Energy Expenditure per Minute (EEM) (kcal/min) & $13.85(1.45)$ \\
\hline Vopendent Variables Final \\
\hline Heart Rate (beats/min) & $27.48(3.64)$ \\
\hline Respiratory Exchange Ratio & $156.46(9.99)$ \\
\hline Energy Expenditure per Minute (EEM) (kcal/min) & $1.13(0.04)$ \\
\hline
\end{tabular}

Means and standard deviations for all continuous descriptive data were calculated. Additionally, frequencies and percentages were determined for categorical descriptive data (Table 2). To determine differences in responses between tasks, a 2-level model with a random intercept, unstructured error structure, maximum likelihood estimation, and between-within method for denominator degrees of freedom was estimated using SAS Proc Mixed. Least squares mean with Tukey's HSD comparison test evaluated differences between specific tasks for all outcome variables (i.e.,
(VO2, RER, HR, and EER), while adjusting for multiple comparisons. Table 3 provides these differences of least squares means adjusted for multiple comparisons, and identifies significant differences at the $\mathrm{p}=.05, .01$, and .001 levels. Group mean values were determined for each of the eight tasks at each time point. Figure 1 provides graphical representations of the eight tasks performed versus each group mean outcome variable (i.e., VO2, RER, HR, and EER) for each of the three time points (i.e., baseline, midpoint, and final).
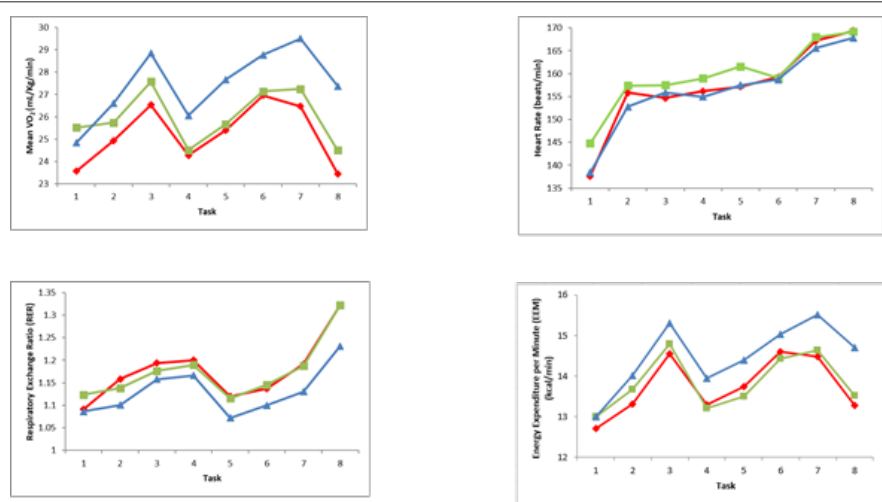

$\rightarrow$ Baseline $\rightarrow$-Midpoint $\leftarrow$-Final

Figure 1: Mean values for outcome variables (VO2, RER, HR, and EEM) at each time point (baseline, midpoint, final) by task (1-8).

Results

Table 3: Differences of Least Squares Means with Tukey's HSD Adjustment.

\begin{tabular}{|c|c|c|c|c|}
\hline CPAT Tasks Compared & VO $_{2}$ & RER & HR & EEM \\
\hline 1,2 & $-1.453^{* * *}$ & -0.033 & $-15.170^{* * *}$ & $-0.760^{* *}$ \\
\hline 1,3 & $-3.328^{* * *}$ & $-0.077^{* * *}$ & $-15.763^{* * *}$ & $-1.970^{* * *}$ \\
\hline 1,4 & -0.694 & $-0.086^{* * *}$ & $-16.494^{* * *}$ & $-0.580^{*}$ \\
\hline 1,5 & $-1.940^{* * *}$ & -0.003 & $-18.495^{* * *}$ & $-0.968^{* * *}$ \\
\hline 1,6 & $-3.315^{* * *}$ & -0.028 & $-18.817^{* * *}$ & $-1.780^{* * *}$ \\
\hline 1,7 & $-3.410^{* * *}$ & $-0.071^{* * *}$ & $-26.745^{* * *}$ & $-1.967^{* * *}$ \\
\hline 1,8 & -0.773 & $-0.193^{* * *}$ & $-28.606^{* * *}$ & $-0.923^{* * *}$ \\
\hline 2,3 & $-1.875^{* * *}$ & $-0.043^{* *}$ & -0.593 & $-1.210^{* * *}$ \\
\hline 2,4 & 0.76 & $-0.053^{* * *}$ & -1.325 & 0.181 \\
\hline
\end{tabular}




\begin{tabular}{|c|c|c|c|c|}
\hline 2,5 & -0.487 & 0.03 & -3.326 & -0.208 \\
\hline 2,6 & $-1.862^{* * *}$ & 0.005 & -3.647 & $-1.020^{* * *}$ \\
\hline 2,7 & $-1.956^{* * *}$ & -0.037 & $-11.575^{* * *}$ & $-1.207^{* * *}$ \\
\hline 2,8 & 0.681 & $-0.160^{* * *}$ & $-13.436^{* * *}$ & -0.163 \\
\hline 3,4 & $2.634^{* * *}$ & -0.009 & -0.732 & $1.390^{* * *}$ \\
\hline 3,5 & $1.388^{* * *}$ & $0.074^{* * *}$ & -2.733 & $1.002^{* * *}$ \\
\hline 3,6 & 0.013 & $0.049^{* *}$ & -3.054 & 0.189 \\
\hline 3,7 & -0.081 & 0.006 & $-10.982^{* * *}$ & 0.002 \\
\hline 3,8 & $2.556^{* * *}$ & $-0.117^{* * *}$ & $-12.843^{* * *}$ & $1.047^{* * *}$ \\
\hline 4,5 & $-1.246^{* *}$ & $0.083^{* * *}$ & -2.001 & -0.389 \\
\hline 4,6 & $-2.621 * * *$ & $0.058^{* * *}$ & -2.323 & $-1.201^{* * *}$ \\
\hline 4,7 & $-2.715^{* * *}$ & 0.015 & $-10.250^{* * *}$ & $-1.388^{* * *}$ \\
\hline 4,8 & -0.079 & $-0.107^{* * *}$ & $-12.111^{* * *}$ & -0.343 \\
\hline 5,6 & $-1.375^{* * *}$ & -0.025 & -0.322 & $-0.812^{* * *}$ \\
\hline 5,7 & $-1.469 * * *$ & $-0.067^{* * *}$ & $-8.249^{* * *}$ & $-1.000^{* * *}$ \\
\hline 5,8 & $1.167^{* *}$ & $-0.190^{* * *}$ & $-10.111^{* * *}$ & 0.046 \\
\hline 6,7 & -0.094 & $-0.043^{*}$ & $-7.928^{* * *}$ & -0.187 \\
\hline 6,8 & $2.543^{* * *}$ & $-0.165^{* * *}$ & $-9.789 * * *$ & $0.858^{* * *}$ \\
\hline 7,8 & $2.637^{* * *}$ & $-0.123^{* * *}$ & -1.861 & $1.045^{* * *}$ \\
\hline
\end{tabular}

Demographic and variable descriptive data are included in Table 2. All 20 participants with complete data were male, with a mean age of $44.1( \pm 6.43)$ years at baseline. Significant differences among VO2, RER, HR, and EEM for comparison among tasks are indicated in Table 2. Among all the comparisons, tasks 2 and 5 are most similar to each other in terms of the outcome variables. Tasks 1 and 3, tasks 1 and 7, tasks 3 and 8, tasks 5 and 7, and tasks 6 and 8 were significantly different from each other for all the outcome variables. Figure 1 shows the mean VO2, RER, HR, and EEM for each task during the baseline (red), midpoint (green), and final (blue) testing times (Figure $1 \&$ Table 3 )

\section{Discussion}

\section{Physiological Measures}

The traditional method of evaluating firefighter's fitness for service is commonly a standardized cardiorespiratory fitness evaluation via a graded exercise test (e.g., VO2 max or Sub-max test such as the Bruce Treadmill Protocol). While this remains a valuable instrument for evaluating both cardiorespiratory fitness and cardiac risk, it does not adequately represent the job-specific physiological demands of firefighting. The CPAT and the modified version we created, on the other hand, appears to represent the net physiological demand as a combination of aerobic and anaerobic challenges, similar to those experienced within a fire-fighting scenario.

This investigation demonstrates the varied physiological demands of the different tasks that compose the CPAT. Because this is a continuous test, the heart rate values steadily increased throughout the test, despite some peaks and valleys in VO2 values, which were much more acutely responsive to task changes. This alone is an interesting finding, as HR and VO2 are often considered to be linearly related and is the concept used to predict VO2 max from submaximal tests [16]. Because VO2 assessment via expired gas analysis is most commonly used during graded exercise testing, and not variable intensity exercise tasks, this linear relationship may not hold true in all circumstances. The traditional CPAT is a continuous and modulating intensity test, and is an entirely different task, in that it allows some degree of recovery during easier tasks, such as with tasks 4 and 8, during which VO2 dropped while HR did not. Additionally, while this group of firefighters did not demonstrate particularly high V02 max values, their training specificity related to their job tasks, near or at lactate threshold, may have resulted in high sustained RER values.

Comparing between the VO2 and RER measurements, there are some unusual features present. First, the average VO2 measurement has a distinct peak during task 3 , whereas the peak in RER is spread out across tasks 3 and 4 . Second, moving from task 4 to 5 results in an increase in average VO2 and a contrasting decrease in RER. Finally, moving from task 7 to 8 results in a decrease in VO2 with a contrasting and drastic increase in RER. This final difference may be skewing the RER measurement when averaged over all tasks. Further investigation of this phenomenon, which was consistent across all 3 time periods, needs to be performed for more information. It would be possible to make similar comparisons with HR as well; however, HR increases consistently with each task. This means that there is likely an exhaustion factor that is confounded with the progression through each task that makes any type of comparison difficult. A new set of data would need to be collected in a way to eliminate this confounding between exhaustion and task in order to make comparisons with HR.

The most notable spikes in VO2 occurred during tasks 3 and 7, in which a heavy weight was carried or dragged. Unlike a standard graded exercise test, which is largely a measurement relative VO2 
max, the introduction of additional weight is a relevant job-specific task that relies upon not only relative VO2 max, but also anaerobic and muscular strength components. This may help explain the apparent lag in the RER responses to the firefighter tasks. RER spikes occur in the tasks immediately following the most strenuous tasks (tasks 4 and 8), despite those tasks having much lower VO2 values. This is likely an effect of delayed $\mathrm{CO} 2$ production associated with bicarbonate buffering of hydrogen ions in response to an anaerobic workload. While RER values were higher than expected considering this was not a maximal test, the incorporation of anaerobic and lifting tasks may be responsible for this observation. Again, these observations demonstrate how different the CPAT is to a graded exercise test in terms of physiological strain. As was described in a previous paper [1], the participants were tested at three time points. At the final time point, VO2 values increased at each task, while RER trended lower, but the relationship between each task remained stable. The increase in VO2 over time for each task reflects an increased work output, as the total number of tasks completed within the 15-minute job task performance increased from an average of 10.72 to 12 , meaning the tasks were completed more quickly.

\section{Practical Applications}

Based on these data, it is recommended that firefighter exercise program design include intervals of work that incorporate continuous cardiorespiratory work with intermittent bouts of high intensity work. High intensity interval training (HIIT) would suit firefighters in this regard, as it employs intermittent bouts of vigorous activity followed by lower intensity periods of recovery [17]. Several studies have demonstrated the effectiveness of HIIT in eliciting cardiorespiratory adaptations when compared to more traditional steady-state endurance training (moderate-intensity continuous training; MICT), citing significantly reduced time commitment and training volume to achieve similar improvements to cardiorespiratory fitness (CRF), as well as increased participant enjoyment [17-19]. Specifically, improvements to CRF resulting from HIIT are attributable to body-wide adaptations that include increased lactate threshold and increased mitochondrial biogenesis and respiration in active skeletal muscle, as well as increased V02max, left ventricular mass, stroke volume, and contractility [19-20]. Further adaptations include increased resting glycogen content, adaptations to peripheral vascular structure and function, and improved exercise performance [17].

Traditional HIIT protocols usually employ large, continuous movements associated with cardiorespiratory exercise, such as cycling, running, stair climbing, or multiple muscle group, body weight resisted exercise. HIIT is especially unique, as it can elicit improvements to muscular strength alongside simultaneous improvements to CRF and presents a potent training stimulus for firefighters that can directly parallel the nature of job-specific tasks. This is clearly exemplified in the CPAT course itself, which in its very nature constitutes HIIT (Table 1). The course consists of eight vigorous job tasks requiring the use of multiple muscle groups to move heavy loads (e.g. tasks 2, 4, and 8), often over long distances (e.g. tasks 1, 3, and 7), separated by intervals of rest (i.e. walking a distance of 85 feet between each station).
Including a similar style of HIIT into firefighter physical conditioning programs could induce adaptations to CRF that could potentially improve job task performance when on a fire or emergency scene. Transitioning firefighter cardiorespiratory exercise from more traditionally practiced MICT to HIIT would result in significant reductions to time commitment and training volume needed to improve markers of CRF. Additionally, incorporating a HIIT style of functional movement training could potentially serve to reduce exercise-related injuries within this population, of which constitute up to a third of injury reports in some municipalities [21]. Functional movement training should address mobilization of the stabilizing muscles along with training supporting muscle groups that facilitate dynamic firefighter job tasks. Lastly, when thinking about the timing of when job demands of firefighters are performed (e.g. under high stress situations and/or middle of the night) and the potential for injury, it is prudent to consider training programs that target pre-habilitation such as functional movement training [22].

\section{Conclusions}

With respect to fitness, designing effective exercise prescriptions to impact firefighter health and longevity is the aim of every municipal, volunteer or wildland fire department. Knowing the actual physical requirement helps us to create exercise programs specific to the demands of the job. Specifically, these data indicate an increase in VO2 over time when performing firefighter job tasks with a lower RER as a result of repeated exposure to performing interval-type job tasks in a continuous or aerobic manner (Figure 1). Therefore, employing dynamic, job-specific exercises through HIIT protocols could stimulate functional training adaptations to muscular strength and aerobic capacity, mimicking the vigorous demands of firefighter job tasks to promote pre-habilitation against on- and off-duty injuries.

\section{Author Contributions}

Authorship is limited to those who have contributed substantially to the work reported. Conceptualization, Dierdra Bycura; Data curation, Dierdra Bycura and Anthony Santos; Formal analysis, Dierdra Bycura and Christopher Ropak, Investigation, Dierdra Bycura and Anthony Santos; Methodology, Dierdra Bycura; Writing - original draft, Dierdra Bycura, Christopher Ropak and Anthony Santos.

\section{Acknowledgments}

The study was funded through a competitive intramural grant program at the sponsoring University to allow for equipment purchase and maintenance, travel expenses and incentives for participants' study participation.

\section{Conflicts of Interest}

The authors declare no conflict of interest.

\section{References}

1. Bycura, DK Dmitrieva, NO, Santos AC, Waugh, et al. (2018) Efficacy of a goal setting and implementation planning intervention on firefighters' cardiorespiratory fitness. Journal of Strength and Conditioning Research pp. 1-11. 
2. Baur DM, Christophi CA, Cook EF, Stefanos N, Kales, et al. (2012) Agerelated decline in cardiorespiratory fitness among career firefighters: Modification by physical activity and adiposity. Journal of Obesity p. 1-6.

3. Baur DM, Christophi CA, Tsismenakis AJ, Cook EF, Kales SN, et al. (2011) Cardiorespiratory fitness predicts cardiovascular risk profiles in career firefighters. J Occup Environ Med 53(10): 1155-1160

4. Jahnke SA, Poston WS, Haddock CK, Jitnarin N (2013) Obesity and incident injury among career firefighters in the central United States. Obesity (Silver Spring) 21(8): 1505-1508.

5. Smith DL, Fehling PC, Frisch A, Haller JM, Winke M, et al. (2012) The prevalence of cardiovascular disease risk factors and obesity in firefighters. J Obes p. 1-9.

6. Storer TW, Dolezal BA, Abrazado ML, Smith DL, Batalin MA, et al. (2014) Firefighter health and fitness assessment: A call to action. J Strength Cond Res 28(3): 661-671.

7. Korre M, Porto LG, Farioli A, Yang J, Christiani DC, et al. (2016) Effect of body mass index on left ventricular mass in career male firefighters. Am J of Cardiol 118(11): 1769-1773.

8. Lentz L, Randall JR, Gross DP, Senthilselvan A, Voaklander D, et al. (2019) The relationship between physical fitness and occupational injury in emergency responders: A systematic review. Am J Ind Med 62(1): 3-13.

9. Gerstner GR, Guiliani K, Mota JA, Ryan ED (2018) Influence of muscle quality on the differences in strength from slow to fast velocities in career firefighters. J Strength Cond Res 32(10): 2982-2986.

10. Gerstner GR, Guiliani HK, Mota JA, Ryan ED (2017) Age related reductions in muscle quality influence the relative difference in strength and power Exp Gerontol 99: 27-34.

11. Candidate Physical Ability Test (CPAT). International Association of Fire Chiefs, 2013. Available at: https://www.iafc.org/topics-and-tools/ safety-health/wellness-fitness-task-force/candidate-physical-abilitytest. Accessed: June 15, 2017.

12. American College of Sports Medicine. ACSM's Guidelines for Exercise Testing and Prescription (10th ed). Philadelphia, PA: Lippincott Williams \& Wilkins, 2018
13. Durand G, Tsismenakis AJ, Jahnke SA, Baur DM, Christophi, et al. (2011) Firefighters' physical activity: Relation to fitness and cardiovascular disease risk. Med Sci Sports Exerc 43(9): 1752-1759.

14. Poston WSC, Haddock CK, Jahnke SA, Jitnarin SA, Day RS, et al. (2013) An examination of the benefits of health promotion programs for the national fire service. BMC Public Health 13: 805.

15. Technical Committee on Fire Service Occupational Safety and Health, National Fire Protection Association. NFPA 1582: Standard on Comprehensive Occupational Medical Program for Fire Departments. Quincy, MA: National Fire Protection Association, 2007.

16. Astrand PO, Ryhming IA (1954) nomogram for calculation of aerobic capacity (physical fitness) from pulse rate during sub-maximal work. J Appl Physiol 7(8): 218-221.

17. Gibala MJ, Little JP, Macdonald MJ, Hawley JA (2012) Physiological adaptations to low-volume, high-intensity interval training in health and disease. J Physiol 590(5): 1077-1084.

18. Gillen JB, ibala (2014) MJ Is high-intensity interval training a timeefficient exercise strategy to improve health and fitness? Appl Physiol Nutr Metab 39(3): 409-412.

19. MacInnis MJ, Gibala MJ (2017) Physiological adaptations to interval training and the role of exercise intensity. J Physiol 595(9): 2915-2930.

20. Wisløff U, Ellingsen $\emptyset$, Kemi OJ (2009) High-intensity interval training to maximize cardiac benefits of exercise training? Exerc Sport Sci Rev 37(3): 139-146.

21. Jahnke SA, Poston WSC, Haddock CK, Jitnarin N (2013) Injury among a population based sample of career fire fighters in the central USA. Inj Prev 19(6): 393-398.

22. Smith DL (2011) Firefighter fitness: Improving performance and preventing injuries and fatalities. Curr Sports Med Rep 10(3): 167-172. 